\title{
NORMALITY AND EXCEPTIONAL FUNCTIONS OF DERIVATIVES
}

\author{
YAN XU \\ (Received 11 November 2002; revised 23 April 2003) \\ Communicated by P. C. Fenton
}

\begin{abstract}
In this paper, we obtain some normality criteria for families of meromorphic functions that concern the exceptional functions of derivatives, which improve and generalize related results of $\mathrm{Gu}$, Yang, Schwick, Wang-Fang, and Pang-Zalcman. Some examples are given to show the sharpness of our results.

2000 Mathematics subject classification: primary 30D35.

Keywords and phrases: meromorphic function, normal family, exceptional functions.
\end{abstract}

\section{Introduction}

Let $G$ be a domain in $\mathbb{C}$, and $\mathscr{F}$ be a family of meromorphic functions defined in $G$. $\mathscr{F}$ is said to be normal in $G$, in the sense of Montel, if for any sequence $f_{n} \in \mathscr{F}$ there exists a subsequence $f_{n_{i}}$, such that $f_{n_{j}}$ converges spherically locally uniformly in $G$, to a meromorphic function or $\infty$ (see $[8,12,20])$.

In 1979, Gu [6] proved the following well-known normality criterion, which was a conjecture of Hayman [9].

THEOREM A. Let $\mathscr{F}$ be a family of meromorphic functions defined in $G$, and let $k$ be a positive integer. If, for every function $f \in \mathscr{F}, f \neq 0, f^{(k)} \neq 1$, then $\mathscr{F}$ is normal in $G$.

This result has undergone various extensions [1, 2, 4, 13, 16, 19]. Yang [18] and Schwick [13] generalized the above theorem and obtained:

The author was partially supported by the NSF of China Grant (Grant 10171047) and NSF of Education Department of Jiangsu Province (03KJB1 10058).

C) 2004 Australian Mathematical Society $1446-7887 / 04 \$ A 2.00+0.00$ 
THEOREM B. Let $\psi \not \equiv 0$ be a analytic function in a domain $G$ and $k \in \mathbb{N}$. Let $\mathscr{F}$ be the family of meromorphic functions in $G$ such that $f$ and $f^{(k)}-\psi$ have no zeros for each $f \in \mathscr{F}$, then $\mathscr{F}$ is normal in $G$.

Wang and Fang [16] generalized Theorem $\mathrm{A}$ by allowing $f$ to have zeros, as follows:

THEOREM C. Let $\mathscr{F}$ be a family of meromorphic functions defined in $G$, and let $k$ be a positive integer. If, for every function $f \in \mathscr{F}, f$ has only zeros of order at least $k+1$ and only poles of order at least 2 , and $f^{(k)} \neq 1$, then $\mathscr{F}$ is normal in $G$.

THEOREM D. Let $\mathscr{F}$ be a family of meromorphic functions defined in $G$, and let $k$ be a positive integer. If, for every function $f \in \mathscr{F}, f$ has only zeros of order at least $k+2$ and $f^{(k)} \neq 1$, then $\mathscr{F}$ is normal in $G$.

If we allow $f$ to have zeros and replace ' 1 ' by a function ' $\psi(z) \not \equiv 0$ ' in Theorem A, does Theorem $A$ still hold? This is a natural but somewhat difficult problem proposed by Y. X. Gu. Recently, Pang and Zalcman [10] proved the following

THEOREM E. Let $\psi(\not \equiv 0)$ be a function holomorphic in a domain $G \subset \mathbb{C}$. Let $\mathscr{F}$ be a family of meromorphic functions defined in $G$, all of whose poles are multiple and whose zeros all have multiplicity at least 3 . If, for every function $f \in \mathscr{F}$, $f^{\prime}(z) \neq \psi(z)$, then $\mathscr{F}$ is normal in $G$.

It is natural to consider the case when $f^{\prime}$ is replaced $f^{(k)}$ in Theorem E. In this paper, we shall prove the following results:

THEOREM 1. Let $\psi(\not \equiv 0)$ be a function holomorphic in a domain $G \subset \mathbb{C}, k \in \mathbb{N}$. Let $\mathscr{F}$ be a family of meromorphic functions defined in $G$, all of whose poles are multiple and whose zeros all have multiplicity at least $k+2$. If, for every function $f \in \mathscr{F}, f^{(k)}(z) \neq \psi(z)$, then $\mathscr{F}$ is normal in $G$.

THEOREM 2. Let $\psi(\neq \equiv)$ be a function holomorphic in a domain $G \subset \mathbb{C}, k$ be a positive integer. Let $\mathscr{F}$ be a family of meromorphic functions defined in $G$, all of whose zeros have multiplicity at least $k+3$. If, for every function $f \in \mathscr{F}, f^{(k)}(z) \neq \psi(z)$ then $\mathscr{F}$ is normal in $G$.

REMARK 1 . The following example shows that the number $k+3$ in Theorem 2 (fo $k=1)$ is best possible. 
EXAMPLE 1 (see [10]). Let $k=1, D=\{z:|z|<1\}$, and $\mathscr{F}=\left\{f_{n}\right\}$, where

$$
f_{n}(z)=\frac{(z-1 / n)^{3}}{z-3 / n}=z^{2}+\frac{3}{n^{2}}+\frac{8}{n^{3}(z-3 / n)} .
$$

Clearly, all zeros of $f_{n}$ have multiplicity 3 . But $\mathscr{F}$ is not normal in $D$. In fact, $f_{n}^{\#}\left(2 / n^{2}\right) \rightarrow \infty$ as $n \rightarrow \infty$, and so by Marty's criterion, $\mathscr{F}$ is not normal in $D$.

THEOREM 3. Let $\psi(\psi \not \equiv 0)$ be a function holomorphic in a domain $G \subset \mathbb{C}, k$ be a positive integer. Let $\mathscr{F}$ be a family of meromorphic functions defined in $G$, all of whose zeros have multiplicity at least $k+2$. If, for every function $f \in \mathscr{F}$, $f^{(k)}(z) \neq \psi(z)$, and $\psi$ has no simple zeros in $G$, then $\mathscr{F}$ is normal in $G$.

REMARK 2. The hypothesis that $\psi$ has no simple zeros in $G$ cannot be omitted in Theorem 3, as is shown by Example 1.

REMARK 3. The following example shows that the exceptional function $\psi(z)$ cannot be extended to the meromorphic case in Theorems 1-3.

EXAMPLE 2. Let $k, l \in \mathbb{N}, D=\{z:|z|<1\}, \psi(z)=1 / z^{k+l}$, and

$$
\mathscr{F}=\left\{f_{n}(z)=1 / n z^{l}, z \in D\right\} \text {. }
$$

Clearly, there exists $n_{0} \in \mathbb{N}$ such that $f_{n}^{(k)}(z)-\psi(z) \neq 0$ for $n \geq n_{0}$. But $\mathscr{F}$ is not normal in $D$.

\section{Some lemmas}

To prove our results, we need the following lemmas.

LEMMA 1 ([16]). Let $f$ be a non-polynomial rational function and $k$ be a positive integer. If $f^{(k)}(z) \neq 1$, then

$$
f(z)=\frac{1}{k !} z^{k}+a_{k-1} z^{k-1}+\cdots+a_{0}+\frac{a}{(z+b)^{m}},
$$

where $a_{k-1}, \ldots, a_{0}, a(\neq 0), b$ are constants and $m$ is a positive integer.

LEMMA 2 ([16]). Let $f$ be a meromorphic function of finite order in the plane, $k$ be a positive integer. If all zeros of $f$ are of order at least $k+2$ and $f^{(k)}(z) \neq 1$, then $f(z)$ is a constant. 
LEMMA 3 ([5]). Let $f$ be a transcendental meromorphic function of finite order and let $b(z)$ be a polynomial which does not vanish identically. If $f$ has only zeros of order at least 2, then $f^{\prime}-b(z)$ has infinitely many zeros.

Here we shall use the standard notation of value distribution theory (see $[7,17])$, $T(r, f), \boldsymbol{m}(r, f), N(r, f), \bar{N}(r, f), \ldots$ We denote by $S(r, f)$ any function satisfying

$$
S(r, f)=o\{T(r, f)\},
$$

as $r \rightarrow \infty$, possibly outside a set with finite measure.

LEMMA 4 ([15]). If $f$ is a transcendental meromorphic function and $k \in \mathbb{N}$, then, for every $\varepsilon>0$,

$$
(k-2) \bar{N}(r, f)+N\left(r, \frac{1}{f}\right) \leq 2 \bar{N}\left(r, \frac{1}{f}\right)+N\left(r, \frac{1}{f^{(k)}}\right)+\varepsilon T(r, f)+S(r, f) .
$$

LEMMA 5. Let $f$ be a transcendental meromorphic function, $k(\geq 2), l$ be positive integers. If $f$ has only zeros of order at least 3 , then $f^{(k)}-z^{l}$ has infinitely many zeros.

Proof. Suppose that $f^{(k)}-z^{l}$ has finitely many zeros. Then

$$
N\left(r, \frac{1}{f^{(k)}-z^{l}}\right)=S(r, f)
$$

By the logarithmic derivative theorem, we have

$$
\begin{aligned}
m(r, & \left.\frac{1}{f}\right)+m\left(r, \frac{1}{f^{(k)}-z^{l}}\right) \\
& \leq m\left(r, \frac{1}{f^{(k+l)}}\right)+m\left(r, \frac{1}{f^{(k+l)}-l !}\right)+S(r, f) \\
& =m\left(r, \frac{1}{f^{(k+l)}}+\frac{1}{f^{(k+l)}-l !}\right)+S(r, f) \\
& \leq m\left(r, \frac{1}{f^{(k+l+1)}}\right)+S(r, f) \\
& \leq T\left(r, f^{(k+l+l)}\right)-N\left(r, \frac{1}{f^{(k+l+1)}}\right)+S(r, f) \\
& \leq T\left(r, f^{(k)}\right)+(l+1) \bar{N}(r, f)-N\left(r, \frac{1}{f^{(k+l+1)}}\right)+S(r, f)
\end{aligned}
$$


Thus

$$
\begin{aligned}
T(r, f) \leq & (l+1) \bar{N}(r, f)+N(r, 1 / f) \\
& +N\left(r, \frac{1}{f^{(k)}-z^{l}}\right)-N\left(r, \frac{1}{f^{(k+l+1)}}\right)+S(r, f) .
\end{aligned}
$$

By Lemma 4 and noting that $k \geq 2$, then, for every $\varepsilon>0$, we have

$$
\begin{aligned}
(l+1) & \bar{N}(r, f)+N(r, 1 / f) \\
& \leq(k+l-1) \bar{N}(r, f)+N(r, 1 / f) \\
& \leq 2 \bar{N}(r, 1 / f)+N\left(r, 1 / f^{(k+l+1)}\right)+\varepsilon T(r, f)+S(r, f) .
\end{aligned}
$$

Combining (2.2) and (2.3), we obtain

$$
T(r, f) \leq 2 \bar{N}\left(r, \frac{1}{f}\right)+N\left(r, \frac{1}{f^{(k)}-z^{l}}\right)+\varepsilon T(r, f)+S(r, f) .
$$

Recalling that the zeros of $f$ are of order $\geq 3$ and setting $\varepsilon=1 / 6$, from (2.1) and (2.4), we get

$$
T(r, f) \leq 6 N\left(r, \frac{1}{f^{(k)}-z^{l}}\right)+S(r, f)=S(r, f),
$$

which contradicts the fact that $f$ is a transcendental meromorphic function. Lemma 5 is proved.

LEMMA 6. (i) Let $k$ be a positive integer, and let $Q(z)$ be a rational function all of whose zeros are of order at least $k+2$ and all of whose poles are multiple with the possible exception of $z=0$. Then, for each positive integer $l, Q^{(k)}(z)=z^{l}$ has a solution in $\mathbb{C}$.

(ii) If all zeros of $Q(z)$ have multiplicity at least $k+3$, then (i) is still valid without the hypothesis on poles of $Q(z)$.

(iii) If $l \neq 1$, then (i) still holds without the hypothesis on poles of $Q(z)$.

PROOF. Fix $l$ and suppose that $Q^{(k)}(z)-z^{l} \neq 0$ for all $z \in \mathbb{C}$. If $Q$ is a polynomial, then $Q^{(k)}(z)=z^{l}+\alpha$, where $\alpha \neq 0$, so that

$$
Q(z)=\frac{l !}{(k+l) !} z^{k+l}+\frac{\alpha}{k !} z^{k}+c_{1} z^{k-1}+c_{2} z^{k-2}+\cdots+c_{k-1},
$$

where $c_{1}, c_{2}, \ldots, c_{k-1}$ are constants. Obviously, $l \neq 1$. Since all zeros of $Q$ have multiplicity at least $k+2$, then $Q^{(k+1)}(z)=Q^{(k)}(z)=\cdots=Q^{\prime}(z)=0$ whenever 
$Q(z)=0$. But $Q^{(k+1)}(z)=l z^{l-1}$ vanishes only for $z=0$. Then $Q(0)=0$, so that $\alpha=Q^{(k)}(0)=0$, a contradiction. Thus $Q$ cannot be a polynomial.

Set

$$
f(z)=Q(z)-\frac{l !}{(k+l) !} z^{k+l}+\frac{1}{k !} z^{k}
$$

Then $f$ is a non-polynomial rational function and $f^{(k)}(z) \neq 1$. By Lemma 1 ,

$$
f(z)=\frac{1}{k !} z^{k}+a_{k-1} z^{k-1}+\cdots+a_{0}+\frac{a}{(z+b)^{m}},
$$

where $a_{k-1}, \ldots, a_{0}, a(\neq 0), b$ are constants and $m$ is a positive integer. Thus

$$
Q(z)=\frac{l !}{(k+l) !} z^{k+l}+a_{k-1} z^{k-1}+\cdots+a_{0}+\frac{a}{(z+b)^{m}} .
$$

There exists a point $z_{0}$ such that $Q\left(z_{0}\right)=0$, and then

$$
\begin{aligned}
Q^{(k)}\left(z_{0}\right) & =z_{0}^{l}+(-1)^{k} \frac{m(m+1) \cdots(m+k-1) a}{\left(z_{0}+b\right)^{m+k}}=0, \\
Q^{(k+1)}\left(z_{0}\right) & =l z_{0}^{l-1}+(-1)^{k+1} \frac{m(m+1) \cdots(m+k) a}{\left(z_{0}+b\right)^{m+k+1}}=0 .
\end{aligned}
$$

Since $a \neq 0$, we know that $z_{0} \neq 0$. Solving for $z_{0}$ from (2.6) and (2.7), we obtain $z_{0}=-b l /(m+k+l)$. Thus $b \neq 0$, and by (2.5),

$$
\mathcal{Q}(z)=\frac{l !(z+b l /(m+k+l))^{m+k+l}}{(k+l) !(z+b)^{m}} .
$$

Again by (2.5), we get

$$
\begin{aligned}
\left(z+\frac{b l}{m+k+l}\right)^{m+k+l} \equiv & z^{k+l}(z+b)^{m}+\frac{(k+l) ! a_{k-1}}{l !} z^{k-1}(z+b)^{m} \\
& +\cdots+\frac{(k+l) ! a_{0}}{l !}(z+b)^{m}+\frac{(k+l) ! a}{l !} .
\end{aligned}
$$

Equating coefficients of $z^{m+k+l-1}$ in (2.9), we obtain $b l=m b$, so that $m=l$ since $b \neq 0$. Since all nonzero poles of $Q$ are multiple, we have $l=m \geq 2$. Then, by equating coefficients of $z^{m+k+1-2}$ in (2.9), we have

$$
\frac{(m+k+l)(m+k+l-1)}{2}\left(\frac{b l}{m+k+l}\right)^{2}=\frac{m(m-1) b^{2}}{2} .
$$

Thus $l=-k$, a contradiction and (i) is proved.

The assumptions in (ii) or (iii) imply that $m=l \geq 2$. As in the proof of (i), we also have (2.10). Then the proofs of (ii) and (iii) are almost the same as the proof of (i). Here we omit the details. 
The well-known Zalcman's lemma is a very important tool in the study of normal families. It has also undergone various extensions and improvements. The following is an up-to-date version, which is due to Pang and Zalcman [11] (see also [2, 3, 16, 21, 22]).

LEMMA 7. Let $k$ be a positive integer and let $\mathscr{F}$ be a family of functions meromorphic in a domain $G$, such that each function $f \in \mathscr{F}$ has only zeros of multiplicity at least $k$, and suppose that there exists $A \geq 1$ such that $\left|f^{(k)}(z)\right| \leq A$ whenever $f(z)=0, f \in \mathscr{F}$. If $\mathscr{F}$ is not normal at $z_{0} \in G$, then, for each $0 \leq \alpha \leq k$, there exist a sequence of points $z_{n} \in G, z_{n} \rightarrow z_{0}$, a sequence of positive numbers $\rho_{n} \rightarrow 0$, and a sequence of functions $f_{n} \in \mathscr{F}$ such that

$$
g_{n}(\zeta)=\frac{f_{n}\left(z_{n}+\rho_{n} \zeta\right)}{\rho_{n}^{\alpha}} \rightarrow g(\zeta)
$$

locally uniformly with respect to the spherical metric, where $g$ is a nonconstant meromorphic function on $\mathbb{C}$, all of whose zeros have multiplicity at least $k$, such that $g^{*}(\zeta) \leq g^{\#}(0)=k A+1$. Moreover, $g$ has order at most 2 .

Here, as usual, $g^{\#}(\zeta)=\left|g^{\prime}(\zeta)\right| /\left(1+|g(\zeta)|^{2}\right)$ is the spherical derivative.

\section{Proof of theorems}

PROOF OF THEOREM 1. Since normality is a local property, without loss of generality, we may assume $G=D=\{z:|z|<1\}$, and

$$
\psi(z)=z^{l}+a_{l+1} z^{l+1}+\cdots=z^{l} \varphi(z), \quad(z \in D),
$$

where $l \geq 1, \varphi(0)=1, \varphi(z) \neq 0$ for $0<|z|<1$, and it is enough to show that $\mathscr{F}$ is normal at each $z \in D$. By Theorem $\mathrm{C}$, we only need to prove that $\mathscr{F}$ is normal at $z=0$.

Consider the family $\mathscr{G}=\{g(z)=f(z) / \psi(z): f \in \mathscr{F}, z \in D\}$. If $f \in \mathscr{F}$, then $f^{(k)}(0) \neq \psi(0)=0$, so that $f(0) \neq 0$ since all zeros of $f$ have multiplicity at least $k+2$. Thus, for any $g \in \mathscr{G}, g(0)=f(0) / \psi(0)=\infty$.

We first prove that $\mathscr{G}$ is normal in $D$. Suppose, on the contrary, that $\mathscr{G}$ is not normal at $z_{0} \in D$. Then by Lemma 7 , there exist a sequence of functions $g_{n} \in \mathscr{G}$, a sequence of complex numbers $z_{n} \rightarrow z_{0}$ and a sequence of positive numbers $\rho_{n} \rightarrow 0$, such that

$$
G_{n}(\zeta)=\frac{g_{n}\left(z_{n}+\rho_{n} \zeta\right)}{\rho_{n}^{k}} \rightarrow G(\zeta)
$$


converges spherically uniformly on compact subsets of $\mathbb{C} . G(\zeta)$ is a nonconstant meromorphic function on $\mathbb{C}$, all zeros of $G(\zeta)$ have multiplicity at least $k+2$, and moreover $G(\zeta)$ is of order at most two.

We distinguish two cases:

Case $1: z_{n} / \rho_{n} \rightarrow \infty$. By simple calculation, we have

$$
g_{n}^{(k)}(z)=\frac{f_{n}^{(k)}(z)}{\psi(z)}-C_{n}^{1} g_{n}^{(k-1)}(z) \frac{\psi^{\prime}(z)}{\psi(z)}-C_{n}^{2} g_{n}^{(k-2)}(z) \frac{\psi^{\prime \prime}(z)}{\psi(z)}-\cdots-g_{n}(z) \frac{\psi^{(k)}(z)}{\psi(z)}
$$

Thus, using notation $\hat{z}_{n}=z_{n}+\rho_{n} \zeta$ for brevity, we have

$$
\begin{aligned}
G_{n}^{(k)}(\zeta)= & g_{n}^{(k)}\left(\hat{z}_{n}\right) \\
= & \frac{f_{n}^{(k)}\left(\hat{z}_{n}\right)}{\psi\left(\hat{z}_{n}\right)}-C_{n}^{l} g_{n}^{(k-1)}\left(\hat{z}_{n}\right) \frac{\psi^{\prime}\left(\hat{z}_{n}\right)}{\psi\left(\hat{z}_{n}\right)}-\cdots-g_{n}\left(\hat{z}_{n}\right) \frac{\psi^{(k)}\left(\hat{z}_{n}\right)}{\psi\left(\hat{z}_{n}\right)} \\
= & \frac{f_{n}^{(k)}\left(\hat{z}_{n}\right)}{\psi\left(\hat{z}_{n}\right)}-C_{n}^{1} g_{n}^{(k-1)}\left(\hat{z}_{n}\right)\left(\frac{l}{\hat{z}_{n}}+\frac{\varphi^{\prime}\left(\hat{z}_{n}\right)}{\varphi\left(\hat{z}_{n}\right)}\right)-\cdots \\
& -g_{n}\left(\hat{z}_{n}\right)\left(\frac{l !}{(l-k) !\left(\hat{z}_{n}\right)^{k}}+C_{k}^{1} \frac{l !}{(l-k+1) !\left(\hat{z}_{n}\right)^{k-1}} \frac{\varphi^{\prime}\left(\hat{z}_{n}\right)}{\varphi\left(\hat{z}_{n}\right)}\right. \\
& \left.+\cdots+\frac{\varphi^{(k)}\left(\hat{z}_{n}\right)}{\varphi\left(\hat{z}_{n}\right)}\right) \\
= & \frac{f_{n}^{(k)}\left(\hat{z}_{n}\right)}{\psi\left(\hat{z}_{n}\right)}-C_{n}^{1} \frac{g_{n}^{(k-1)}\left(\hat{z}_{n}\right)}{\rho_{n}}\left(\frac{l \rho_{n}}{\hat{z}_{n}}+\frac{\rho_{n} \varphi^{\prime}\left(\hat{z}_{n}\right)}{\varphi\left(\hat{z}_{n}\right)}\right)-\cdots \\
& -\frac{g_{n}\left(\hat{z}_{n}\right)}{\rho_{n}^{k}}\left(\frac{l ! \rho_{n}^{k}}{(l-k) !\left(\hat{z}_{n}\right)^{k}}+C_{k}^{1} \frac{l \rho_{n}^{k-1}}{(l-k+1) !\left(\hat{z}_{n}\right)^{k-1}} \frac{\rho_{n} \varphi^{\prime}\left(\hat{z}_{n}\right)}{\varphi\left(\hat{z}_{n}\right)}\right. \\
& \left.+\cdots+\frac{\rho_{n}^{k} \varphi^{(k)}\left(\hat{z}_{n}\right)}{\varphi\left(\hat{z}_{n}\right)}\right) .
\end{aligned}
$$

On the other hand, we have $\lim _{n \rightarrow \infty}\left(\rho_{n} / \hat{z}_{n}\right)=0$ and

$$
\lim _{n \rightarrow \infty} \frac{\rho_{n} \varphi^{(i)}\left(\hat{z}_{n}\right)}{\varphi\left(\hat{z}_{n}\right)}=0, \quad(i=1,2, \ldots, k)
$$

uniformly on compact subsets of $\mathbb{C}$. Therefore, on every compact subsets of $\mathbb{C}$ which contains no poles of $G(\zeta)$, we have

$$
\frac{f_{n}^{(k)}\left(\hat{z}_{n}\right)}{\psi\left(\hat{z}_{n}\right)} \rightarrow G^{(k)}(\zeta)
$$

Since $f_{n}^{(k)}(z) / \psi(z) \neq 1$, by Hurwitz's theorem, we know that either $G^{(k)}(\zeta) \neq 1$ or $G^{(k)}(\zeta) \equiv 1$ for any $\zeta \in \mathbb{C}$ that is not a pole of $G(\zeta)$. Clearly, these also hold for 
all $\zeta \in \mathbb{C}$. If $G^{(k)}(\zeta) \neq 1$, then by Lemma $2, G(\zeta)$ is a constant, a contradiction. If $G^{(k)}(\zeta) \equiv 1$, then

$$
G(\zeta)=\frac{1}{k !} \zeta^{k}+c_{k-1} \zeta^{k-1}+\cdots+c_{0}
$$

which contradicts the fact that all zeros of $G(\zeta)$ have multiplicity at least $k+2$.

Case 2: $z_{n} / \rho_{n} \rightarrow \alpha$, a finite complex number. Then

$$
\frac{g_{n}\left(\rho_{n} \zeta\right)}{\rho_{n}^{k}}=\frac{g_{n}\left(z_{n}+\rho_{n}\left(\zeta-z_{n} / \rho_{n}\right)\right)}{\rho_{n}^{k}}=G_{n}\left(\zeta-z_{n} / \rho_{n}\right) \rightarrow G(\zeta-\alpha)=\widetilde{G}(\zeta),
$$

spherically uniformly on compact subsets of $\mathbb{C}$. Clearly, all zeros of $\widetilde{G}(\zeta)$ have multiplicity at least $k+2$, and $\zeta=0$ is a pole of $\widetilde{G}(\zeta)$ with multiplicity at least $l$, and the other poles of $\widetilde{G}(\zeta)$ are multiple.

Set $H_{n}(\zeta)=f_{n}\left(\rho_{n} \zeta\right) / \rho_{n}^{k+l}$. Then

$$
H_{n}(\zeta)=\frac{\psi\left(\rho_{n} \zeta\right)}{\rho_{n}^{l}} \frac{f_{n}\left(\rho_{n} \zeta\right)}{\rho_{n}^{k} \psi\left(\rho_{n} \zeta\right)}=\frac{\psi\left(\rho_{n} \zeta\right)}{\rho_{n}^{l}} \frac{g_{n}\left(\rho_{n} \zeta\right)}{\rho_{n}^{k}} .
$$

Note that $\lim _{n \rightarrow \infty} \psi\left(\rho_{n} \zeta\right) / \rho_{n}^{l}=\zeta^{l}$ uniformly on compact subsets of $\mathbb{C}$, thus

$$
H_{n}(\zeta) \rightarrow \zeta^{l} \widetilde{G}(\zeta)=H(\zeta)
$$

uniformly on compact subsets of $\mathbb{C}$. Obviously, all zeros of $H(\zeta)$ have multiplicity at least $k+2$, and all nonzero poles of $H(\zeta)$ are multiple, and $H(0) \neq 0$ since $\widetilde{G}$ has a pole of order at least $l$ at $\zeta=0$. We also have

$$
H_{n}^{(k)}(\zeta)-\frac{\psi\left(\rho_{n} \zeta\right)}{\rho_{n}^{l}} \rightarrow H^{(k)}(\zeta)-\zeta^{l}
$$

uniformly on every compact subset of $\mathbb{C}$ which contains no pole of $\widetilde{G}$.

ClaIM. $H^{(k)}(\zeta) \neq \zeta^{l}$

Otherwise there exists $\zeta_{0}$ such that $H^{(k)}\left(\zeta_{0}\right)=\zeta_{0}^{l}$. Then $H$ is holomorphic at $\zeta_{0}$. We consider two subcases.

Case 2.1: $\zeta_{0} \neq 0$. Since

$$
H_{n}^{(k)}(\zeta)-\frac{\psi\left(\rho_{n} \zeta\right)}{\rho_{n}^{l}}=\frac{f_{n}^{(k)}\left(\rho_{n} \zeta\right)-\psi\left(\rho_{n} \zeta\right)}{\rho_{n}^{l}} \neq 0,
$$

then Hurwitz's theorem implies that $H^{(k)}(\zeta) \equiv \zeta^{l}$. Thus

$$
H(\zeta)=\frac{\zeta^{k+l} l !}{(l+k) !}+a_{1} \zeta^{k-1}+a_{2} \zeta^{k-2}+\cdots+a_{k}
$$


where $a_{1}, a_{2}, \ldots, a_{k}$ are constants, and

$$
H^{(k-1)}(\zeta)=\frac{\zeta^{l+1}}{l+1}+(k-1) ! a_{1} .
$$

Since all zeros of $H(\zeta)$ have multiplicity at least $k+2$, then $H^{(k-1)}(\zeta)$ must have a zero with multiplicity at least 4 . Hence $a_{1}=0$. Similarly, we can deduce that $a_{2}=a_{3}=$ $\cdots=a_{k}=0$. It follows that $H(\zeta)=\zeta^{k+l} l ! /(l+k)$ !. Then $\widetilde{G}(\zeta)=\zeta^{k} l ! /(l+k)$ !, which contradicts the fact that all zeros of $\widetilde{G}(\zeta)$ have multiplicity at least $k+2$.

Case 2.2: $\zeta_{0}=0$. Then $H_{n}(\zeta)$ is holomorphic and $H_{n}(\zeta) \rightarrow H(\zeta)$ uniformly on a neighbourhood of 0 . Indeed, $H(\zeta)$ is holomorphic at 0 , so $\widetilde{G}(\zeta)$ has a pole of exact order $l$ at 0 . On the other hand, for each $n$, the pole of $g_{n}\left(\rho_{n} \zeta\right)$ at 0 has also exact order $l$. Then, $\zeta=0$ is the zero of $1 / \widetilde{G}(\zeta)$ and $1 / g_{n}\left(\rho_{n} \zeta\right)$ is of order $l$. Note that since $g_{n}\left(\rho_{n} \zeta\right) / \rho_{n}^{k} \rightarrow \widetilde{G}(\zeta)$, spherically uniformly on compact subsets of $\mathbb{C}$, there exist a positive integer $n_{0}$ and $r>0$ such that

$$
\left|\frac{\rho_{n}^{k}}{g_{n}\left(\rho_{n} \zeta\right)}-\frac{1}{\widetilde{G}(\zeta)}\right| \leq \frac{1}{|\widetilde{G}(\zeta)|}
$$

for all $n \geq n_{0}$ and each $\zeta \in\{\zeta:|\zeta|=r\}$. By Rouché's theorem, $1 / g_{n}\left(\rho_{n} \zeta\right)$ has no zeros in $D_{r}^{\prime}=\{\zeta: 0<|\zeta|<r\}$ for $n \geq n_{0}$, and then $g_{n}\left(\rho_{n} \zeta\right)$ has no poles in $D_{r}^{\prime}$ for $n \geq n_{0}$. Thus $H_{n}(\zeta)$ is holomorphic in $D_{r}^{\prime}$, and $H_{n}(\zeta) \rightarrow H(\zeta)$ uniformly on a neighbourhood of 0 . Hence, the same argument as in Case 2.1 also applies for Case 2.2.

Now, we have $H^{(k)}(\zeta) \neq \zeta^{l}$. By Lemma 3 (for $k=1$ ) and Lemma 5 (i) (for $k \geq 2$ ), $H(\zeta)$ must be a rational function. However, Lemma 7 shows that $H^{(k)}(\zeta)=\zeta^{l}$ has a solution in $\mathbb{C}$, a contradiction. We have proved that $\mathscr{G}$ is normal on $D$.

It remains to show that $\mathscr{F}$ is normal at $z=0$. Since $\mathscr{G}$ is normal in $D$, then the family $\mathscr{G}$ is equicontinuous in $D$ with respect to the spherical distance. On the other hand, $g(0)=\infty$ for each $g \in \mathscr{G}$, so there exists $\delta>0$ such that $|g(z)| \geq 1$ for all $g \in \mathscr{G}$ and each $z \in D_{\delta}=\{z:|z|<\delta\}$. It follows that $f(z) \neq 0$ for all $f \in \mathscr{F}$ and $z \in D_{\delta}$. Suppose that $\mathscr{F}$ is not normal at $z=0$. Since $\mathscr{F}$ is normal in $0<|z|<1$, the family $1 / \mathscr{F}=\{1 / f: f \in \mathscr{F}\}$ is holomorphic in $D_{\delta}$ and normal in $D_{\delta}^{\prime}=\{z: 0<|z|<\delta\}$, but it is not normal at $z=0$. Thus there exists a sequence $\left\{1 / f_{n}\right\} \subset 1 / \mathscr{F}$ which converges locally uniformly in $D_{\delta}^{\prime}$, but not in $D_{\delta}$. The maximum modulus principle implies that $1 / f_{n} \rightarrow \infty$ in $D_{\delta}^{\prime}$. Thus $f_{n} \rightarrow 0$ converges locally uniformly in $D_{\delta}^{\prime}$, and hence so does $\left\{g_{n}\right\} \subset \mathscr{G}$, where $g_{n}=f_{n} / \psi$. But $\left|g_{n}(z)\right| \geq 1$ for $z \in D_{\delta}$, a contradiction. This completes the proof of Theorem 1 .

PROOF OF THEOREM 2 AND THEOREM 3 . Theorem 2 and Theorem 3 can be proved by using the same argument as in Theorem 1. The main difference is in using Lemma 6 (ii) (to prove Theorem 2) or Lemma 6 (iii) (to prove Theorem 3). We here omit the details. 


\section{Acknowledgement}

We wish to thank Professors H. H. Chen and M. L. Fang for valuable suggestions.

\section{References}

[1] W. Bergweiler, 'Normality and exceptional values of derivatives', Proc. Amer. Math. Soc. 129 (2000), 121-129.

[2] H. H. Chen, 'Yosida function and Picard values of integral functions and their derivatives', Bull. Austral. Math. Soc. 54 (1996), 373-381.

[3] H. H. Chen and Y. X. Gu, 'An improvement of Marty's criterion and its applications', Sci. China, Ser. A 36 (1993), 674-681.

[4] M. L. Fang, 'A note on a problem of Hayman', Analysis 20 (2000), 45-49.

[5] —-, 'Picard values and normality criterion', Bull. Korean Math. Soc. 38 (2001), 379-387.

[6] Y. X. Gu, 'A normal criterion of meromorphic families', Scientia Sinica, Math. Issue 1 (1979), 267-274.

[7] W. K. Hayman, 'Picard values of meromorphic functions and their derivatives', Ann. of Math. (2) 70 (1959), 9-42.

[8] - Meromorphic functions (Clarendon Press, Oxford, 1964).

[9] - Research problems in function theory (Athlone Press, London, 1967).

[10] X. C. Pang and L. Zalcman, 'Normal families of meromorphic functions with multiple zeros and poles', Israel J. Math. 136 (2003), 1-9.

[11] _ _ 'Normal families and shared values', Bull. London Math. Soc. 18 (1995), 437-450.

[12] J. Schiff, Normal families (Springer, New York, 1993).

[13] W. Schwick, 'Exceptional functions and normality', Bull. London Math. Soc. 29 (1997), 425-432.

[14] —_, 'On Hayman's alternative for families of meromorphic functions', Complex Variables Theory Appl. 32 (1997), 51-57.

[15] Y. F. Wang, 'On Mues conjecture and Picard values', Sci. China 36 (1993), 28-35.

[16] Y. F. Wang and M. L. Fang, 'Picard values and normal families of meromorphic functions with multiple zeros', Acta Math. Sinica (N.S.) 14 (1998), 17-26.

[17] Y. Xu and M. L. Fang, 'On normal families of meromorphic functions', J. Aust. Math. Soc. 74 (2003), 155-164.

[18] L. Yang, 'Normal families and fixed-points of meromorphic functions', Indiana Univ. Math. J. 35 (1986), 179-191.

[19] _ _ 'Normality of families of meromorphic functions', Sci. China 9 (1986), 898-908.

[20] - Value distribution theory (Springer, Berlin; Science Press, Beijing, 1993).

[21] L. Zalcman, 'A heuristic principle in complex function theory', Amer. Math. Monthly 82 (1975), 813-817.

[22] —_, Normal families: new perspectives', Bull. Amer. Math. Soc. 35 (1998), 215-230.

\section{Department of Mathematics}

Nanjing Normal University

Nanjing 210097

P. R. China

e-mail: xuyan@njnu.edu.cn 
J. Aust. Math. Soc. 76 (2004) 